\title{
Processing Accuracy of Instantaneous Values of a Stochastic Signal in an Inertial Measurement System
}

\author{
Adam Kowalczyk, Rafał Chorzępa \\ Rzeszów University of Technology, Faculty of Electrical and Computer Engineering, 12 Powstańców Warszawy Ave., \\ 35-959 Rzeszów,Poland,kowadam@prz.edu.pl rchorz@prz.edu.pl
}

\begin{abstract}
The article presents an analysis of the dynamic error occurring when processing a stochastic signal in an inertial measurement system. The problem was illustrated using both a calculation and a laboratory example. The technique of conditional averaging of signals was used in the experiment. The possibility to minimize the root mean square value of the error as well as the need for a time correction of measurement values in an inertial measurement system was demonstrated.
\end{abstract}

Keywords: Stochastic signal, distortion, delay, dynamic error.

\section{INTRODUCTION}

The primary task during measurement processing of physical random signals is to minimize the original distortions of signals at their points of reception and in the analogue pre-processing system [1]-[3]. Another important task in the stochastic signal measurement process is to minimize random processing errors of complex analogue and analogue-digital measurement processing systems [4][7]. Primary measurement transducers (sensors) are critical to the processing accuracy of instantaneous values of a stochastic signal. No signal distortion check in the preprocessing track prevents further correct measurement processing.

In analogue processing of randomly variable physical signals, distortions of instantaneous values of signals may be caused either by outside interferences or by actual imperfect characteristics of processing systems. An example of a distortion system is an inertial measurement transducer (e.g., a temperature sensor). In measuring the instantaneous value, an inertial system introduces distortion dependent on the time constant $T$. The output signal of the inertial transducer is distorted and delayed in relation to the input signal. Instantaneous values of the output signal recorded in real time do not represent the actual instantaneous values of the measured parameter [1], [2], [3].

\section{DYNAMIC ERROR}

General dynamic processing in an inertial measurement system (Fig.1.) can be described by impulse response:

$$
k(t)= \begin{cases}\frac{k}{T} e^{-\frac{\tau}{T}} & \text { for } \tau \geq 0, \\ 0 & \text { for } \tau<0,\end{cases}
$$

and spectral transmittance:

$$
K(j \omega)=\frac{k}{1+j \omega T}
$$

where: $k-$ is the static gain; $T-$ is the time constant.

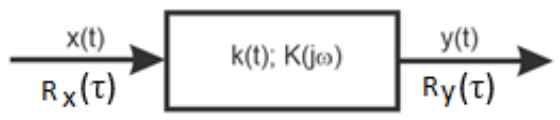

Fig.1. Model of dynamic processing.

The processing quality of the stochastic signal $x(t)$ in a measurement system is expressed by the root mean square value of the error:

$$
\overleftarrow{\Delta^{2}}(t)=\overline{[x(t)-y(t)]^{2}}
$$

where: $x(t)$ - the processed physical stochastic input signal; $y(t)$ - the output signal of the measurement system.

If the signal $x(t)$ is stationary, then the error value $\overline{\Delta^{2}}(t)$ does not depend on time and is determined by error variance:

$$
\overline{\Delta^{2}}=\operatorname{Var}(\Delta)=\sigma_{x}^{2}+\sigma_{y}^{2}-2 R_{x y}(0),
$$


where: $\sigma_{x}^{2}$ and $\sigma_{y}^{2}-$ variances of signals $x(t)$ and $y(t)$; $R_{x y}(0)$ - correlation function of signals $x(t)$ and $y(t)$ for $\tau=$ 0 .

Practical tasks of random signal processing concern the signals which are not theoretical white noise. Actual signals, most often low-band ones, do not have a flat characteristic of the power spectral density function. In practice, such signals occur after the passing of broadband noises through inertial systems. A simple and practically useful model is one with low-band noise with an exponential autocorrelation function. After passing through another inertial system, such a signal has a finite value of the autocorrelation function derivative $R_{y}(\tau)$ for $\tau=0$. When a random signal passes through inertial systems, its probability density function becomes normalized [8], [9], [10].

For an inertial model of processing a stochastic signal $x(t)$ with a limited bandwidth, exponentially correlated with the correlation interval $\tau_{k}=\frac{1}{\alpha}$, the autocorrelation function $R_{x}(\tau)$ is expressed by:

$$
R_{x}(\tau)=\sigma_{x}^{2} e^{-\alpha|\tau|},
$$

The corresponding double-sided power spectral density is expressed by the following relationship:

$$
S_{x}(\omega)=\frac{2 \sigma_{x}^{2} \alpha}{\alpha^{2}+\omega^{2}} .
$$

Fig.2. graphs the functions $R_{x}(\tau)$ and $S_{x}(\omega)$.

a)

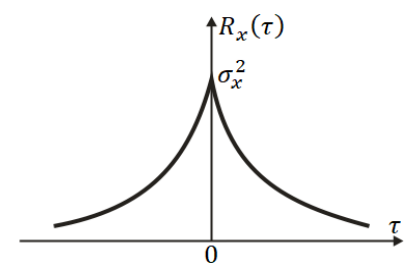

b)

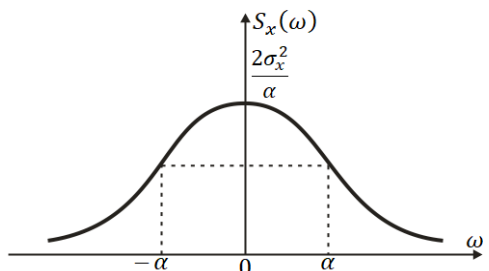

Fig.2. Characteristics of the autocorrelation function a) and the double-sided spectral density power b).

In order to calculate the error variance (4), one must first calculate the correlation functions $R_{y}(\tau)$ and $R_{x y}(\tau)$ :

$$
\begin{aligned}
& R_{y}(\tau)=E[y(t) y(t+\tau)], \\
& R_{x y}(\tau)=E[x(t) y(t+\tau)] .
\end{aligned}
$$

After indirect substitutions, transformations and calculations, one can demonstrate that for the time constant $T=\frac{1}{\beta}$ and for $k=1$ in an inertial measurement system, the autocorrelation function $R_{y}(\tau)$ and power spectral density $S_{y}(\omega)$ of the output signal are expressed as follows:

$$
\begin{gathered}
R_{y}(\tau)=\frac{\beta \sigma_{x}^{2}}{\alpha^{2}-\beta^{2}}\{\alpha \exp (-\beta|\tau|)-\beta \exp (-\alpha|\tau|)\} \\
S_{y}(\omega)=\frac{2 \beta^{2} \alpha \sigma_{x}^{2}}{\left(\alpha^{2}+\omega^{2}\right)\left(\beta^{2}+\omega^{2}\right)}
\end{gathered}
$$

Based on the relationship (8),

$$
\sigma_{y}^{2}=R_{y}(0)=\frac{\beta \sigma_{x}^{2}}{\alpha+\beta}
$$

Using a convolution integral combining random signals $x(t)$ and $y(t)$ of a linear system with an impulse response $\mathrm{k}(\mathrm{t})$, the relationship (7b) can be transformed to the following form:

$$
\begin{gathered}
R_{x y}(\tau)=E\left[x(t) \int_{0}^{\infty} x(t+\tau-\lambda) k(\lambda) d \lambda\right] \\
=\int_{0}^{\infty} R_{x}(\tau-\lambda) k(\lambda) d x .
\end{gathered}
$$

For $\tau \geq 0$ and the relationships (2), (5):

$$
\begin{gathered}
R_{x y}(\tau)=k \cdot \beta \sigma_{x}^{2}\left[\frac{e^{-\beta \tau}-e^{-\alpha \tau}}{\alpha-\beta}+\frac{e^{-\beta \tau}}{\alpha+\beta}\right], \\
R_{x y}(0)=\frac{k \cdot \beta \sigma_{x}^{2}}{\alpha+\beta} .
\end{gathered}
$$

The calculated error variance (4) for $k=1$ equals:

$$
\overline{\Delta^{2}}=\sigma_{x}^{2}+\frac{\beta \sigma_{x}^{2}}{\alpha+\beta}-2 \frac{\beta \sigma_{x}^{2}}{\alpha+\beta}=\sigma_{x}^{2} \frac{\alpha}{\alpha+\beta}=\sigma_{x}^{2} \frac{\frac{T}{\tau_{k}}}{1+\frac{T}{\tau_{k}}}
$$

while the relation between the error variance and the signal variance $\sigma_{x}^{2}$ is expressed by the relationship:

$$
\overline{\overline{\Delta^{2}}}=\frac{\frac{T}{\sigma_{x}^{2}}}{1+\frac{T}{\tau_{K}}} .
$$

The relationship between $\frac{\overline{\Delta^{2}}}{\sigma_{x}^{2}}$ and $\frac{T}{\tau_{K}}$ is presented in Fig.3.

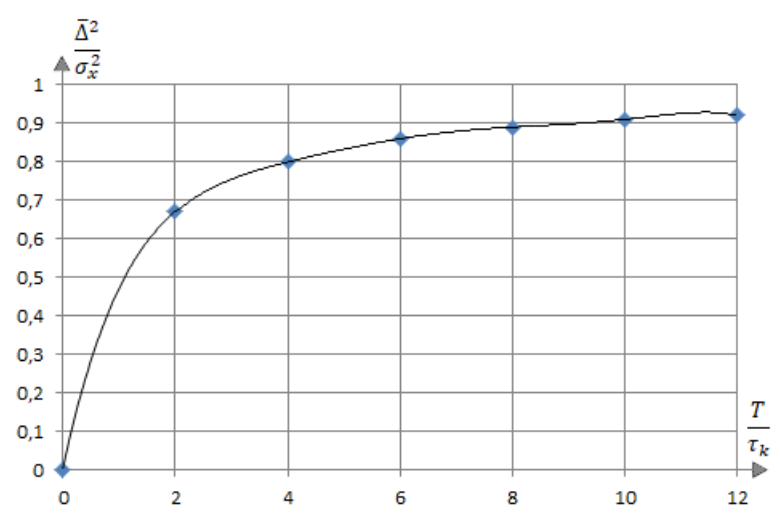

Fig.3. The relationship between $\frac{\overline{\Delta^{2}}}{\sigma_{x}^{2}}$ and $\frac{T}{\tau_{K}}$. 


\section{MINIMIZING THE VALUE OF THE ROOT MEAN SQUARE ERROR}

Frequency characteristics, both concerning the amplitude and phase of the inertial system, show that in addition to smoothing distortions, the signal $y(t)$ ) at the output of the inertial system is delayed relative to the input signal $x(t)$. An important metrological question is to determine what instantaneous values of the input signal $x(t)$ one should refer to the instantaneous values $y(t)$ obtained as the output of the inertial measurement system, along with processing error evaluation in an inertial system.

The delayed signal $y(t)$ at the output of the inertial system can be compared with the value of the signal $x(t)$ by calculating the difference:

$$
\Delta(t)=x(t)-y(t+\tau)
$$

The root mean square value of the error does not depend on the current time $t$, but on the delay $\tau$ :

$$
\overline{\Delta^{2}}=\sigma_{x}^{2}+\sigma_{y}^{2}-2 R_{x y}(\tau)
$$

In order to determine the minimum condition of relationship (17), such a value of $\tau=\tau_{\text {opt }}$. must be found for which the function $R_{x y}(\tau)$ is maximum.

As specified in Section 2, the distributions of real random low-band signals passing through inertial systems are gradually normalized. It is reasonable to assume the normality of processed signals $x(t)$ and $y(t)$.

For normal distributions, the cross correlation function $R_{x y}(\tau)$ can be expressed using a more easily experimentally determined function of the conditional expected value of the signal $y(t+\tau)$ with the condition that $x(t)=x_{p}$ imposed on the input signal $x(t)$ [11], [12].

$$
E\left[\left.y(t+\tau)\right|_{x(t)=x_{p}}\right]=\mu_{y \mid x_{p}}(\tau)=\frac{R_{x y}(\tau)}{\sigma_{x}^{2}} x_{p}
$$

In a similar way one can create the normalized autocorrelation functions $\rho_{x}(\tau)$ and $\rho_{y}(\tau)$ from the relationship for the conditional expected values of individual signals $x(t)$ and $y(t)$

$$
\begin{aligned}
& E\left[\left.x(t+\tau)\right|_{x(t)=x_{p}}\right]=x_{p} \rho_{x}(\tau), \\
& E\left[\left.y(t+\tau)\right|_{y(t)=y_{p}}\right]=y_{p} \rho_{y}(\tau) .
\end{aligned}
$$

When using the relationship (18), the equation (17) will then take the following form:

$$
\overline{\Delta^{2}}=\sigma_{x}^{2}+\sigma_{y}^{2}-2 \frac{\sigma_{x}^{2}}{x_{p}} \mu_{\left.y\right|_{x_{p}}}(\tau)
$$

The function $\mu_{y_{\mid x_{p}}}$ for the graph of $\mathrm{x}(\mathrm{t})$ with exponential autocorrelation (5) processed in an inertial system with the time constant $T=\frac{1}{\beta}$, on the basis of (12) and (18), is described by the following relationship:

$$
\mu_{y_{\mid x_{p}}}(\tau)=k \beta x_{p}\left[\frac{e^{-\beta \tau}-e^{-\alpha \tau}}{\alpha-\beta}-\frac{e^{-\beta \tau}}{\alpha+\beta}\right] .
$$

When determining the maximum of the function $\mu_{y_{\mid x_{p}}}(\tau)$ the following condition is arrived at:

$$
\frac{\tau_{o p t}}{\tau_{K}}=\frac{\frac{T}{\tau_{K}}}{1-\frac{T}{\tau_{K}}} \ln \frac{2}{\frac{T}{\tau_{K}}+1} .
$$

Values of $\tau_{\text {opt }}$ calculated from the expression (23) indicate that for the minimum root mean square value of the processing error in an inertial system, the current values of the signal on the input of the system should be related to the respective future values of the processed signal at the output, shifted by the time $\tau_{\text {opt }}$.

Fig.4. shows the relationship between $\frac{\tau_{o p t}}{\tau_{K}}$ and $\frac{T}{\tau_{K}}$.

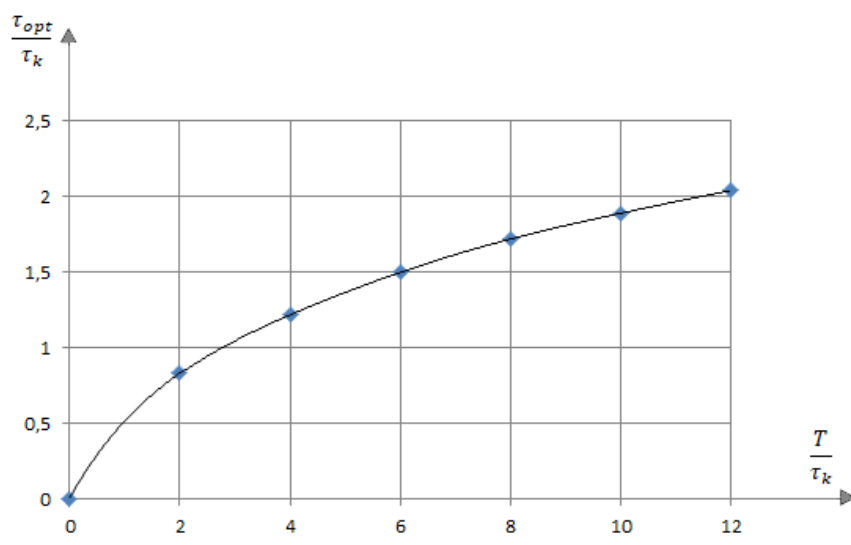

Fig.4. The relationship between $\frac{\tau_{o p t}}{\tau_{K}}$ and $\frac{T}{\tau_{K}}$.

With the values of $\sigma_{x}, k, \alpha, \beta$, and $\tau_{o p t}$ assumed and known, or designated experimentally, the minimum value of the root mean square error variance can be calculated from the relationship (21):

$$
\overline{\Delta_{m i n}^{2}}=\sigma_{x}^{2}\left[\frac{\alpha+2 \beta}{\alpha+\beta}-\frac{2 \mu_{y \mid x_{p}}\left(\tau_{o p t}\right)}{x_{p}}\right] .
$$

\section{EXPERIMENTAL EXAMPLE}

The optimal delay value of the reading $\tau$ opt of the output signal in an inertial system processing a random variable input signal $x(\mathrm{t})$ with a normal distribution $N\left(x: 0, \sigma_{\mathrm{x}}\right)$ was calculated for the time constant of the transducer $T=$ $1.114 \mathrm{~ms}$ and the relation $\frac{T}{\tau_{K}}=10.82$. A measurement experiment was carried out for the calculation data. The results of the calculations and measurements were then compared.

Based on the relationship (23)

$$
\begin{gathered}
\tau_{\text {opt }}=\frac{T}{1-\frac{T}{\tau_{K}}} \ln \frac{2}{\frac{T}{\tau_{K}}+1}= \\
=\frac{1.114 \cdot 10^{-3}}{1-10.82} \ln \frac{2}{10.82+1}=201 \cdot 10^{-3} \mathrm{~ms} .
\end{gathered}
$$


Fig.5. to Fig.8. show the results of the experiment. In the inertial system with the time constant $t=1.11410^{-6} \mathrm{~s}$, gain $\mathrm{k}=3$ occurred. The parameters used in experimental models were: $\alpha=9708$ and $\beta=897$. The results of the experiment (Fig.6. and Fig.8.) confirm the theoretically calculated optimal time delay value of the output reading $t_{0}=t+$ $201 \cdot 10^{3} \mathrm{~s}$. At the moment of time $t$, the signal $y(t)$ is better described by the earlier behavior of the original signal $x(t-\tau)$. Minimizing the root mean square reconstruction error of the signal $x(t)$, with the behavior of $y(t)$ recorded in time, one must properly account for the behavior values of $y(t+$ $\tau_{\text {opt }}$ ) shifted on the graph in time by $201 \mathrm{~ms}$ to the right (Fig.8.).

For $\sigma_{x}=0.5 \mathrm{~V}$ and $\tau_{o p t}=201 \cdot 10^{-3} \mathrm{~ms}, \quad \overline{\Delta_{\min }^{2}}=$ $0.42 \sigma_{x}^{2}=0.105 \mathrm{~V}^{2}$ was calculated, which is approximately $46 \%$ variance of the error calculated from the relationship (14).

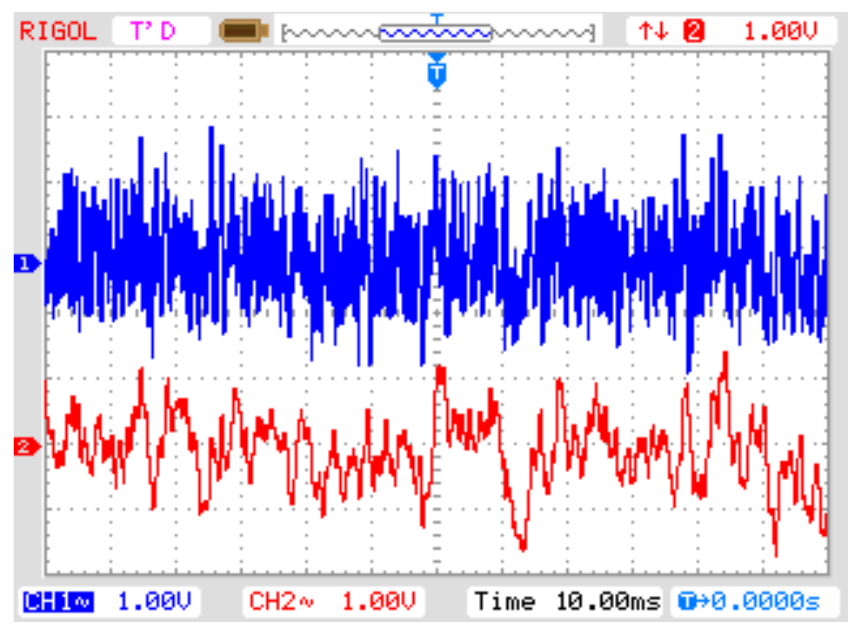

Fig.5. Graphs of the signals: $1-x(t) ; 2-y(t)$.

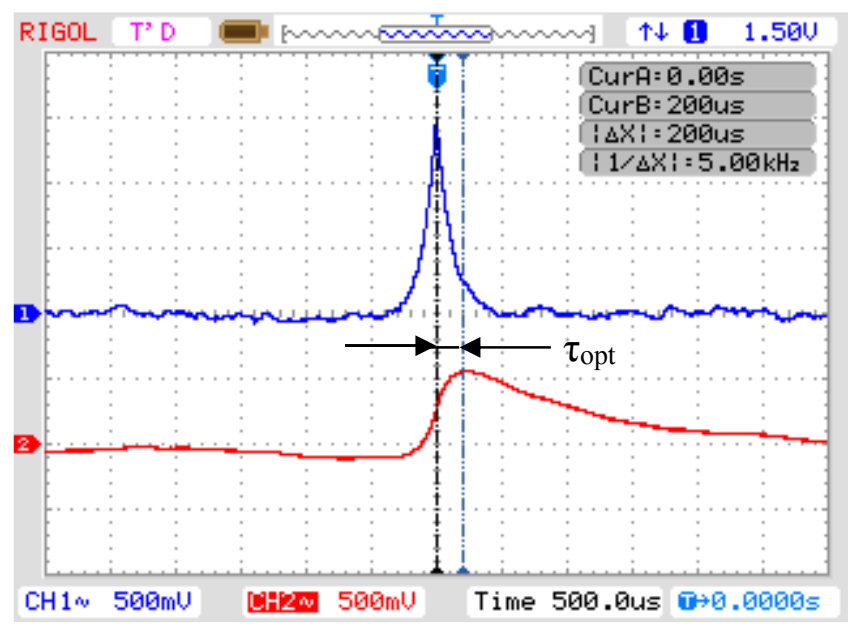

Fig.6. Characteristics of conditional mean values:

1 - characteristics of the conditional expected value $\mu_{x_{\mid x_{p}}}(\tau)$ proportional to $\rho_{x}(\tau)$;

2 - characteristics of the conditional expected value $\mu_{y_{\mid x_{p}}}(\tau)$ proportional to $\rho_{x y}(\tau) ; \tau_{o p t}$ - optimum delay value of the signal $y(t)$.

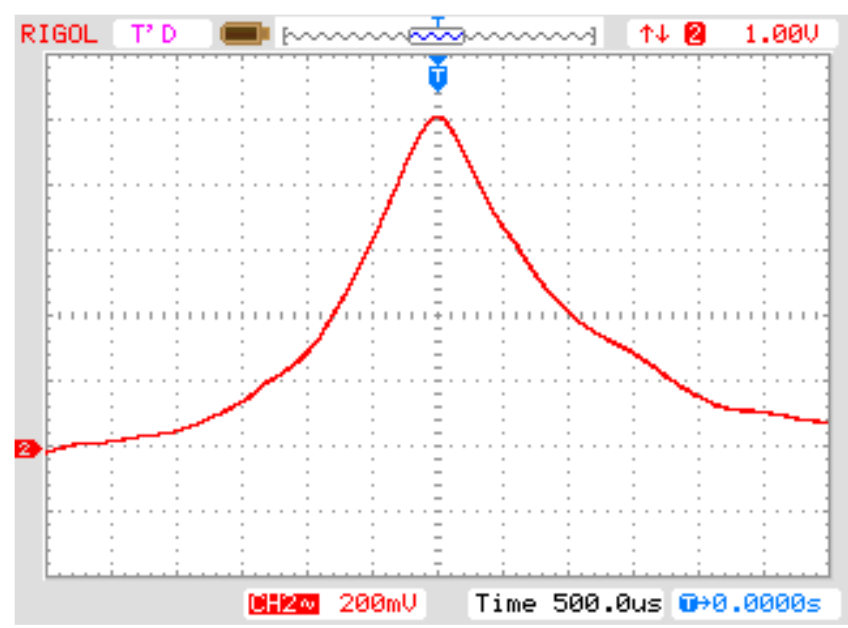

Fig.7. Characteristics of the conditional average value $\mu_{y_{\mid y_{p}}}(\tau)$ proportional to $\rho_{y}(\tau)$.

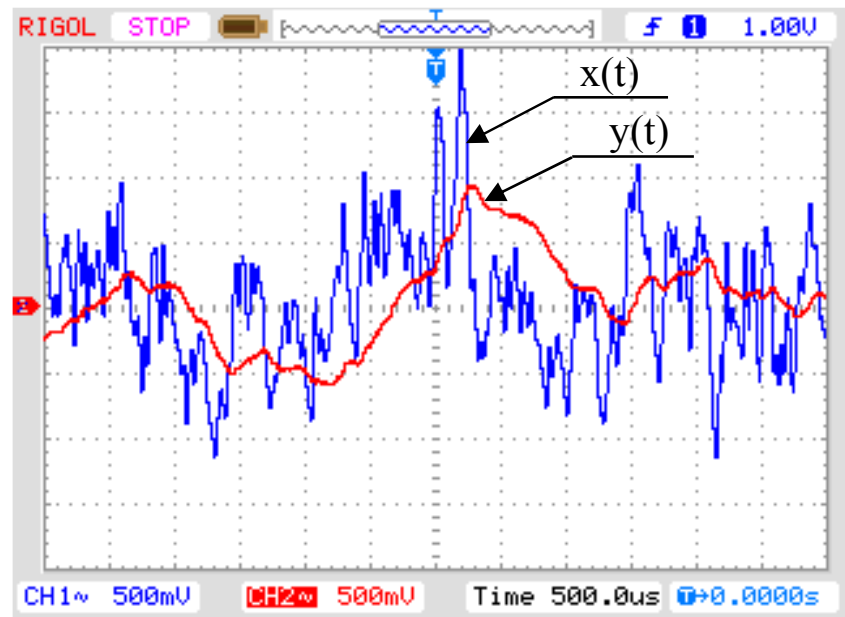

Fig.8. Graphs of signals: 1 - input signal $x(t) ; 2$ - output signal $y(t)$, delayed and distorted.

\section{CONCLUSIONS}

1) The white noise which is used in theoretical models of low pass filtration and random signal processing is not very useful as a model in practical applications. In such situations, one may use noises with limited frequency bands, such as low-band noises with a virtually exponential autocorrelation function and a finite value of the autocorrelation function derivative for $\tau=0$.

2) In processing of random low-band signal, the processing systems are often inertial systems with frequency bands which are significantly narrower than the band of the processed input noise.

3) In addition to smoothing distortions, the signal $y(t)$ at the output of the inertial system is delayed relative to the input signal $x(t)$. Earlier behavior at the time $t$ of the original signal $x(t)$ better describes the signal $y(t+\tau)$.

4) Knowing the values of the correlation interval $\tau_{k}$ of the input signal $x(t)$ exponentially correlated, and the time constant $T$ of the inertial system, one can determine the 
optimal value $\tau_{\text {opt }}$ of shifting the reading moments recorded in time of the output signal value $y\left(t+\tau_{o p t .}\right)$.

5) Signal delays and dynamic errors, which are crucial in measurements, depend on the time constant of inertial systems, which in practice may vary from several dozen $\mathrm{ms}$ to a few minutes for temperature sensors. For the cited example of an experiment, the optimal delay equaled $18 \%$ of the value of the time constant $T$ of the inertial system.

\section{ACKNOWLEDGMENT}

This work was partially supported by Rzeszów University of Technology under project UPB.EM.20.001 and by Polish Ministry of Science and Higher Education under the program "Regional Initiative of Excellence" in 2019 - 2022, project number 027/RID/2018/19, funding amount 11999 900 PLN.

\section{REFERENCES}

[1] Hagel, R., Zakrzewski, J. (1984). Miernictwo dynamiczne (Dynamic Measurement). Warsaw, Poland: WNT.

[2] Granovskij, V.A. (1984). Динамические измерения (Dynamic Measurements). Leningrad, Russia: Energoatomizdat.

[3] Woschni, E.G. (1972). Messdynamik (Dynamic Measurement). Leipzig, Germany: Hirzel Verlag.

[4] Vujicic, V. (2001). Generalized low-frequency stochastic true RMS instrument. IEEE Transactions on Instrumentation and Measurement, 50 (5), 1089-1092.
[5] Sovilj, P.M., Milovancev, S.S., Vujicic, V. (2011). Digital stochastic measurement of a nonstationary signal with an example of EEG signal measurement. IEEE Transactions on Instrumentation and Measurement, 60 (9), 3230-3232.

[6] Kamenský, M., Kováč, K. (2011). Correction of ADC errors by additive iterative method with dithering. Measurement Science Review, 11 (1), 15-18.

[7] Pjevalica, V., Pjevalica, N., Kastelan, I., Petrovic, N. (2018). Acceleration of digital stochastic measurement simulation based on concurrent programming. Elektronika ir Elektrotechnika, 24 (6), 21-27.

[8] Bendat, J.S., Piersol, A.G. (2000). Random Data Analysis and Measurement Procedures. Wiley.

[9] Papoulis, A. (1972). Prawdopodobieństwo, zmienne losowe $i$ procesy stochastyczne (Probability, Random Variables, and Stochastic Processes). Warsaw, Poland: WNT.

[10] Shanmugan, K.S., Breipohl, A.M. (1988). Random Signals Detection, Estimation and Data Analysis. Wiley.

[11] Kowalczyk, A., Szlachta, A., Hanus, R, Chorzepa, R. (2017). Estimation of conditional expected value for exponentially autocorrelated data. Metrology and Measurement Systems, 24 (1), 69-78.

[12] Kowalczyk, A. (2015). Pomiarowe zastosowania warunkowego uśredniania sygnałów. Rzeszów, Poland: Politechnika Rzeszowska, ISBN 978-83-7934011-8.

Received March 3, 2020 Accepted August 5, 2020 